\title{
X-ray standing wave enhanced scattering from mesoporous silica thin films
}

Cite as: Appl. Phys. Lett. 110, 041603 (2017); https://doi.org/10.1063/1.4974922

Submitted: 05 November 2016 . Accepted: 12 January 2017. Published Online: 24 January 2017

Longlong Wu, Yupu Liu, Xiao Wang, Geng Wang, Dongyuan Zhao, and Gang Chen
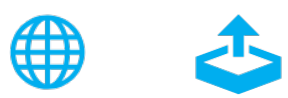

\section{ARTICLES YOU MAY BE INTERESTED IN}

Experimental evidence for $\mathrm{x}$-ray standing wave modulated surface scattering effect Applied Physics Letters 114, 141601 (2019); https://doi.org/10.1063/1.5085231

Broadening microwave absorption via a multi-domain structure APL Materials 5, 046104 (2017); https://doi.org/10.1063/1.4979975

X-ray and optical characterizations of DNA-mediated Janus nanostructures Applied Physics Letters 109, 233101 (2016); https://doi.org/10.1063/1.4971355

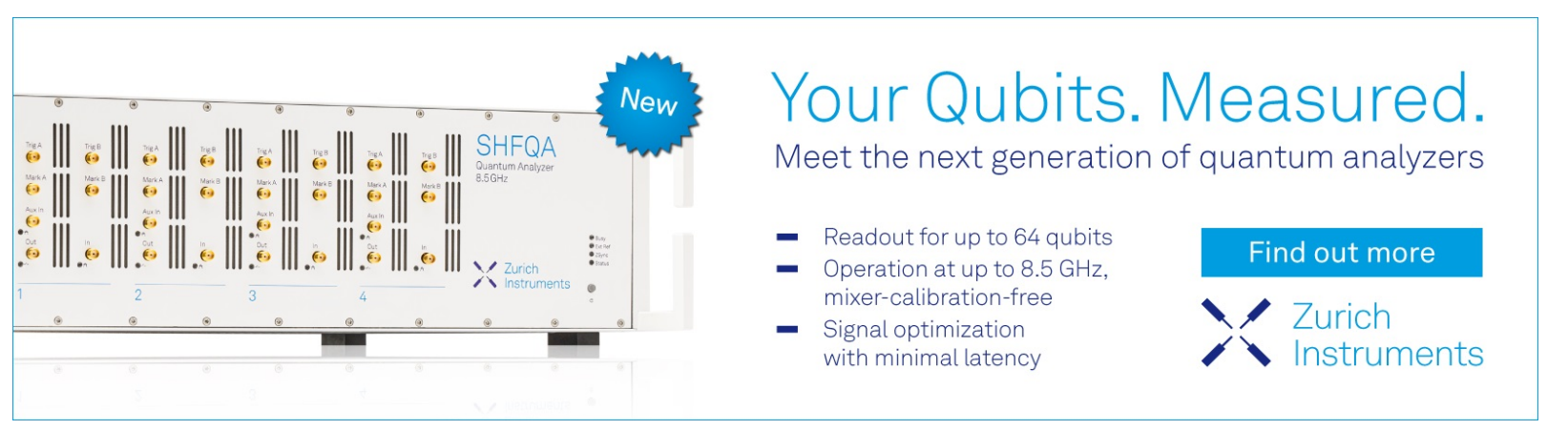




\title{
X-ray standing wave enhanced scattering from mesoporous silica thin films
}

\author{
Longlong Wu, ${ }^{1,2,3, a)}$ Yupu Liu, ${ }^{4, a)}$ Xiao Wang, ${ }^{1}$ Geng Wang, ${ }^{5}$ Dongyuan Zhao, ${ }^{4}$ \\ and Gang Chen ${ }^{1,2,3,5, b)}$ \\ ${ }^{1}$ School of Physical Science and Technology, ShanghaiTech University, Shanghai 201210, China \\ ${ }^{2}$ Shanghai Institute of Microsystem and Information Technology, Chinese Academy of Sciences, \\ Shanghai 200050, China \\ ${ }^{3}$ University of Chinese Academy of Sciences, No. 19A Yuquan Road, Beijing 100049, China \\ ${ }^{4}$ Department of Chemistry, Laboratory of Advanced Materials, Fudan University, Shanghai 200433, China \\ ${ }^{5}$ Shanghai Synchrotron Radiation Facility, Shanghai Institute of Applied Physics, Chinese Academy of \\ Sciences, Shanghai 201204, China
}

(Received 5 November 2016; accepted 12 January 2017; published online 24 January 2017)

\begin{abstract}
X-ray standing wave enhanced scattering effects are observed in mesoporous silica thin films (MSTFs) deposited on rough indium tin oxide coated glass substrates in grazing-incidence smallangle $\mathrm{x}$-ray scattering studies. The distorted-wave Born approximation theory along with a rigorous wave function analysis is employed to elucidate the dynamical scattering processes occurring in MSTFs. The data analysis is significantly simplified by expressing the x-ray scattering intensity from MSTFs in two separate functions of the wave vector transfer, where the in-plane correlation between neighboring mesopore channels is decoupled from the out-of-plane interference interaction. Remarkable agreement is achieved between experiment and theory. Published by AIP Publishing.

[http://dx.doi.org/10.1063/1.4974922]
\end{abstract}

Mesoporous films have attracted much attention due to their extensive applications in optics, electronics, sensing, and catalysis. $^{1-3}$ Among them, mesoporous silica thin films (MSTFs), stable over a broad range of temperatures and $\mathrm{pH}$ values, can provide versatile platforms for many applications, such as catalyst supports and biomedical carriers. MSTFs with mesopore channels (mesochannels) aligned vertically with respect to their surfaces are difficult to fabricate but particularly desirable, because they offer transport channels for electrons, ions, and molecules, which are suited to solar cell, fuel cell, and membrane separation practices. ${ }^{4-6}$ The physical properties of mesoporous films are largely dependent on their mesopore structure and morphology, e.g., size, shape, orientation, and ordering. In general, transmission electron microscopy (TEM), scanning electron microscopy (SEM), atomic force microscopy (AFM), and x-ray scattering and diffraction techniques have been employed to determine the physical parameters of mesoporous films. These characterization techniques have their own merits and drawbacks and are complementary to one another. For thin films, grazing-incidence small-angle X-ray scattering (GISAXS) is a powerful tool for elucidating the structure, morphology, and ordering at nanometer scale and also capable of providing statistical information and probing buried objects. ${ }^{7}$ In addition, x-ray standing waves (XSWs) were generated at glancing angles in organic films deposited on high-density flat substrates and exploited as a sensitive probe to characterize the internal structure of thin films. ${ }^{8-15}$ Besides density and thickness constraints, the two unstated requirements for XSWs are an ultra-smooth substrate (or an X-ray mirror) and a uniform thin film. It is not obvious that an XSW can be formed inside a porous film sitting on a rough substrate. Herein, synchrotron-based GISAXS

\footnotetext{
${ }^{\text {a) }} \mathrm{L}$. Wu and Y. Liu contributed equally to this work.

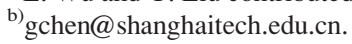

and X-ray reflectivity (XRR) techniques are employed to study the structure and dynamical scattering processes of MSTFs containing vertically aligned mesochannels. XSW-enhanced scattering effects are observed from a mesoporous film deposited on a rugged indium tin oxide (ITO) glass substrate. The optical process and the electric field intensity (EFI) distribution in each layer are evaluated through a rigorous wave function analysis, while the multiple scattering effect is accounted by means of the distorted-wave Born approximation (DWBA) theory.

The MSTF samples were prepared via the oil-water biphase stratification approach. The detailed synthesis procedure will be reported elsewhere. GISAXS and XRR measurements were carried out at the beamlines BL16B and BL14B of Shanghai Synchrotron Radiation Facility (SSRF), respectively, with the incident $\mathrm{x}$-ray photon energy of 10 $\mathrm{keV}$ (wavelength $\lambda=1.24 \AA$ ). Two-dimensional GISAXS patterns were collected using a Mar165 CCD detector with the pixel size of $80 \mu \mathrm{m} \times 80 \mu \mathrm{m}$ and the sample-to-detector distance was set at $1860 \mathrm{~mm}$. The experimental setup and scattering geometry are schematically shown in Fig. 1(a). The mesochannels are aligned vertically to the surface of the MSTF that stays conformed to the contour of the ITO substrate as shown by the TEM and SEM images in Fig. 1(b). There are visible crystal grains in the ITO substrate and its surface roughness is about $2.5 \mathrm{~nm}$ as shown by the SEM and AFM images in Fig. 1(c). The thickness of MSTF is around $30 \mathrm{~nm}$ and that of ITO is about $180 \mathrm{~nm}$.

As is known, the critical angles for total external reflection of silica, ITO, and glass at photon energy of $10 \mathrm{keV}$ are $0.173^{\circ}, 0.296^{\circ}$, and $0.172^{\circ}$, respectively. Since the critical angle of ITO is larger than that of silica, XSWs could be generated inside the MSTF layer of the MSTF/ITO/glass system. ${ }^{8}$ In order to investigate its X-ray scattering property, a series of GISAXS measurements were conducted by continuously 


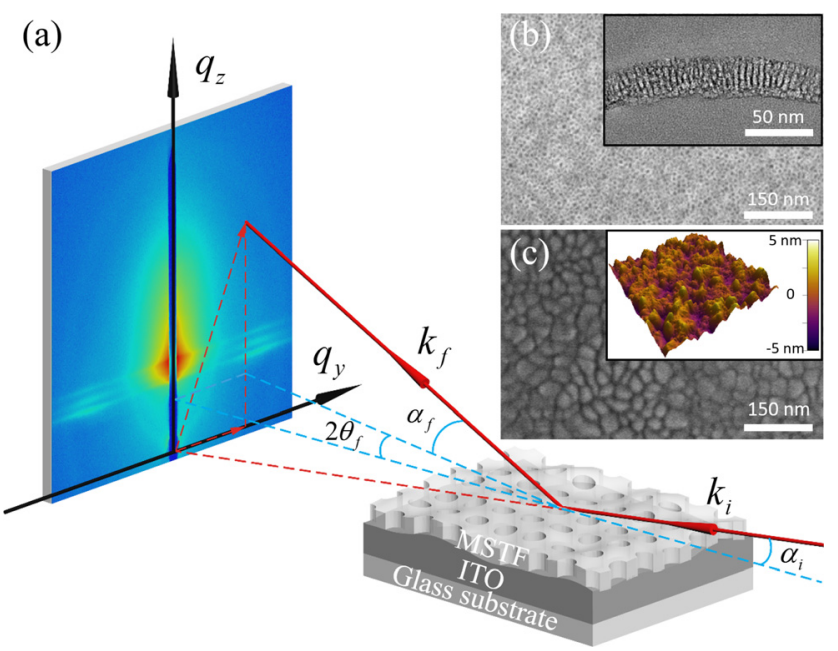

FIG. 1. (a) The schematic diagram of the GISAXS geometry. The incident and scattered wave vectors are $k_{i}$ and $k_{f}, \alpha_{i}$ is the incident angle of x-ray beam, $2 \theta_{f}$ and $\alpha_{f}$ are the azimuthal and exit scattering angles. (b) SEM image of the MSTF/ITO/glass sample. The inset is the cross-sectional TEM image of a free-standing MSTF film. (c) SEM image of the pretreated ITO glass substrate. The AFM image (an area of $1 \mu \mathrm{m} \times 1 \mu \mathrm{m}$ ) is shown in the inset.

varying the incident angle $\alpha_{i}$ at an increment of $0.02^{\circ}$ (supplementary material). The typical scattering patterns taken at $\alpha_{i}$ of $0.04^{\circ}, 0.08^{\circ}, 0.12^{\circ}, 0.18^{\circ}, 0.22^{\circ}, 0.26^{\circ}, 0.30^{\circ}$, $0.40^{\circ}$, and $0.50^{\circ}$ are shown in Figs. 2(a)-2(h), respectively. For $\alpha_{i}=0.04^{\circ}$, well below the critical angle of silica, there are two side lobes with interference fringes and a weak scattering ring R1 passing through them as shown in Fig. 2(a). As $\alpha_{i}$ increases, the diffuse scattering in the region between two side lobes is intensified and a new scattering ring (R2) appears at large angles as shown in Figs. 2(b) and 2(c). For $\alpha_{i}=0.18^{\circ}$, higher than the critical angle of silica and lower than that of ITO, scattering mainly comes from the MSTF layer. Compared to those in Figs. 2(a)-2(c), the scattering pattern alters notably, where the fringe configuration of the side lobes changes and the intensity modulation along the $q_{z}$

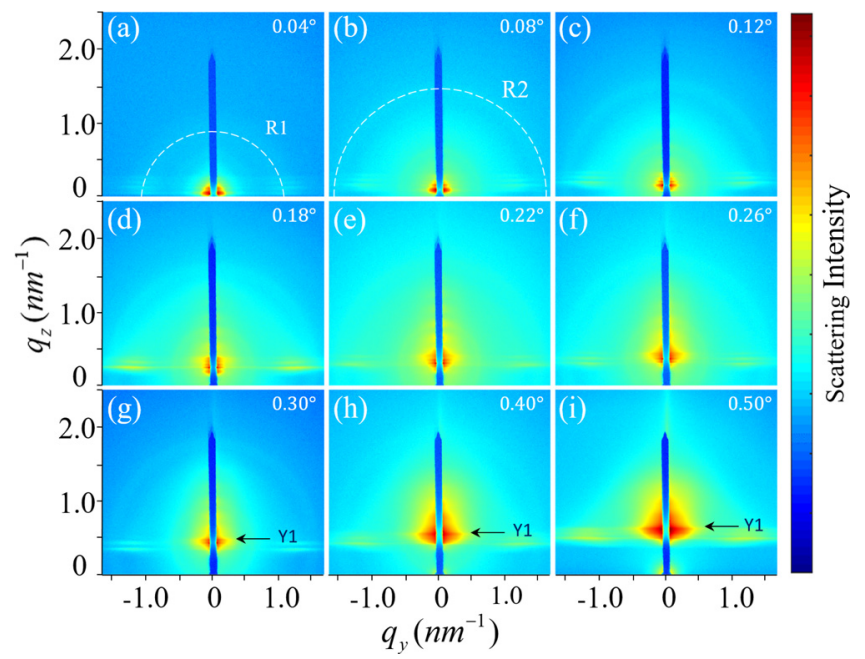

FIG. 2. Representative GISAXS patterns taken at incident angles of (a) $0.04^{\circ}$, (b) $0.08^{\circ}$, (c) $0.12^{\circ}$, (d) $0.18^{\circ}$, (e) $0.22^{\circ}$, (f) $0.26^{\circ}$, (g) $0.30^{\circ}$, (h) $0.40^{\circ}$, and (i) $0.50^{\circ}$. R1 and $\mathrm{R} 2$ are two scattering rings marked for easy identification. Y1 is the Yoneda peak of ITO. direction near the beamstop becomes visible. Further increasing the incident angle $\alpha_{i}$, as shown in Figs. 2(e) and 2(f), the side lobe profiles are almost faded into the diffuse scattering background, while scattering intensifies in the central region near the beamstop and the intensity modulation along $q_{z}$ is clearly visible. For $\alpha_{i}=0.3^{\circ}$, at about the critical angle of ITO, x-rays start to penetrate through both the MSTF and ITO layers and enter the glass substrate. At this stage, the diffuse scattering background from the MSTF/ITO interface reduces dramatically, the double-fringe structure of the side lobes is salient, and the scattering ring R2 comes into sight again, as shown in Fig. 2(g). When the incident angle $\alpha_{i}$ is increased to $0.40^{\circ}$ and $0.50^{\circ}$ as shown in Figs. 2(h) and 2(i), the scattering feature in the central region, mainly from the ITO film, dominates the whole image and the Yoneda peak of ITO (Y1) emerges above the horizon of the side lobes that are still perceptible from the diffuse scattering background.

The intensity evolution of R2 as a function of the incident angle is one of the salient features on the scattering patterns shown in Fig. 2. To find its physical origin, GISAXS measurements were carried out on ITO-coated glass substrates. The second-order scattering ring owing to crystal grains in ITO films ${ }^{16,17}$ occurs at the same wave vector transfer value as R2 is detected. Close examinations reveal that $\mathrm{R} 2$ is centered around the peak positions of the reflected beams. Besides R2, there are multifold x-ray scattering effects encountered in the MSTF/ITO/glass system, including surface-enhanced scattering near the critical angle of total external reflection, XSW-enhanced scattering from the MSTF, and surface roughness and interference scattering effects due to the layered structure. Moreover, the size, shape, and ordering of the MSTF mesochannels bring additional features to the GISAXS patterns. To elucidate the underlying scattering mechanism and trace their physical origins, the transmission, reflection, resonance, and interference interactions for each layer are evaluated through a rigorous wave function analysis by imposing proper boundary conditions at each interface. As shown in the inset of Fig. 3(a), including the top air layer, the ITO glass supported MSTF sample can be divided into four layers with different optical constants, labeled as $j=0,1,2$, and 3 for air, MSTF, ITO, and glass, respectively. When x-rays impinge on the MSTF surface, the amplitude of electromagnetic field satisfies the stationary wave equations, and their wave functions can be expressed as

$$
\Psi^{(0)}(\boldsymbol{r}, \boldsymbol{k})=e^{i \boldsymbol{k}_{\|} \cdot \boldsymbol{r}_{\|}} \begin{cases}e^{-i k_{z, 0} z}+R_{0} e^{i k_{z, 0} z}, & z>0 \\ T_{1} e^{-i k_{z, 1} z}+R_{1} e^{i k_{z, 1} z}, & 0 \geq z>z_{1} \\ T_{2} e^{-i k_{z, 2} z}+R_{2} e^{i k_{z, 2} z}, & z_{1} \geq z>z_{2} \\ T_{3} e^{-i k_{z, 3} z}, & z_{2} \geq z,\end{cases}
$$

for an incoming plane x-ray wave with $\boldsymbol{k}=\left(k_{\|}, k_{z, j}\right)$ and $k_{z, j}=-\sqrt{k_{j}^{2}-k_{\|}^{2}}$. The $k_{z, j}$ are the vertical components of the wave vectors and $R_{j}$ and $T_{j}$ are the wave function amplitudes for $j=0,1,2$, or 3 . The wave functions describing x-ray propagation in the MSTF/ITO/glass system can be solved through differential equations or a matrix formalism. ${ }^{18}$ The resultant expressions for $R_{j}$ and $T_{j}$ are given as 

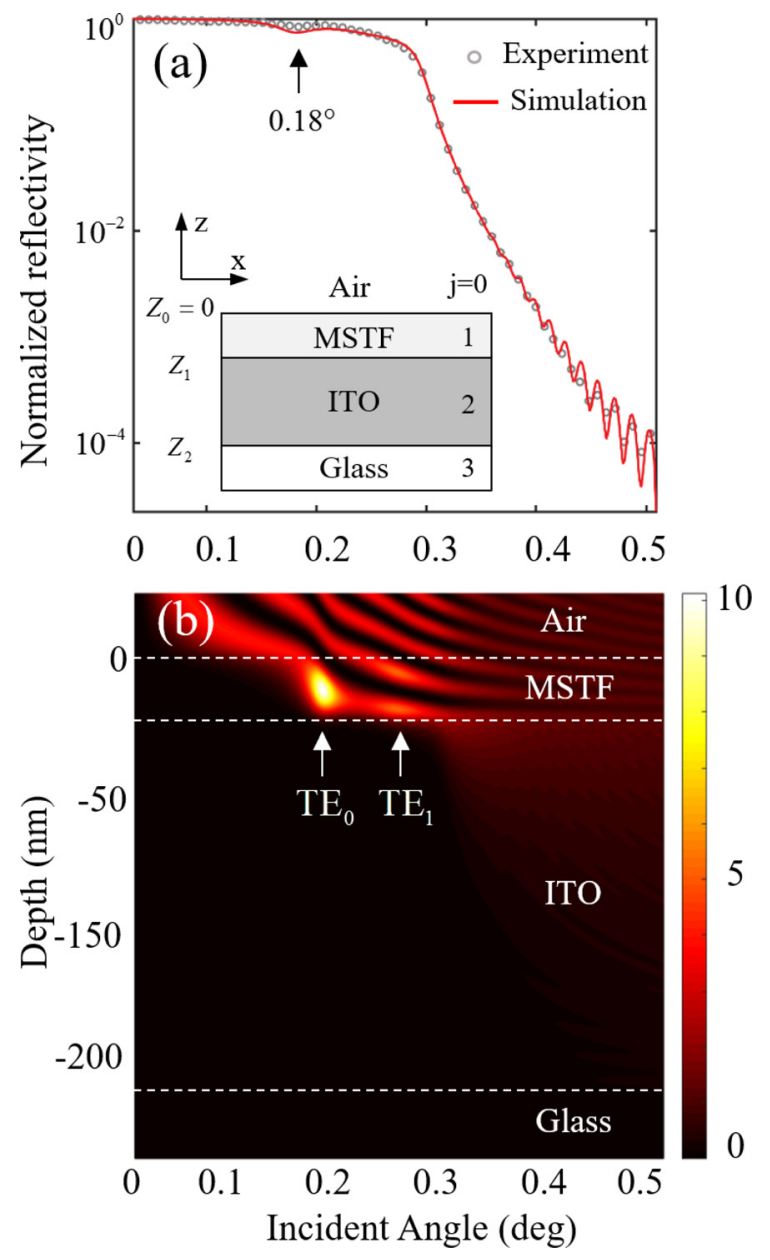

FIG. 3. (a) The normalized experimental XRR curve (black circles) and the simulated curve (red line) obtained using the reflectivity coefficient $R_{0}$. (b) The two-dimensional map of the normalized EFI distribution plotted as functions of the X-ray penetration depth and the incident angle $\alpha_{i}$, using the parameters obtained from the XRR fitting.

$$
\begin{gathered}
R_{0}=\eta\left(r_{0,1}+r_{0,1} r_{1,2} r_{2,3} e^{-2 i k_{z, 2} h_{2}}\right. \\
\left.\quad+r_{1,2} e^{-2 i k_{z, 1} h_{1}}+r_{2,3} e^{-2 i\left(k_{z, 1} h_{1}+k_{z, 2} h_{2}\right)}\right) \\
R_{1}=\eta\left(t_{0,1} r_{1,2} e^{-2 i k_{z, 1} h_{1}}\right. \\
\left.\quad+t_{0,1} r_{2,3} e^{-2 i\left(k_{z, 1} h_{1}+k_{z, 2} h_{2}\right)}\right) \\
T_{1}=\eta\left(t_{0,1}+t_{0,1} r_{1,2} r_{2,3} e^{-2 i k_{z, 2} h_{2}}\right) \\
R_{2}=\eta t_{0,1} t_{1,2} t_{2,3} e^{-i\left(k_{z, 1} h_{1}+2 k_{z, 2} h_{2}\right)} \\
T_{2}=\eta t_{0,1} t_{1,2} e^{-i k_{z, 1} h_{1}} \\
T_{3}=\eta t_{0,1} t_{1,2} t_{2,3} e^{-i\left(k_{z, 1} h_{1}+k_{z, 2} h_{2}\right)}
\end{gathered}
$$

where $\quad \eta=1 /\left[1+r_{0,1} r_{1,2} e^{-2 i k_{z, 1} h_{1}}+r_{0,1} r_{2,3} e^{-2 i\left(k_{z, 1} h_{1}+k_{z, 2} h_{2}\right)}\right.$ $\left.+r_{1,2} r_{2,3} e^{-2 i k_{z, 2} h_{2}}\right]$. The $r_{j, j+1}$ and $t_{j, j+1}$ are the Fresnel reflection and transmission coefficients for the interface between the $j$ th and $(j+1)$ th layers, whose expressions are given as $r_{j, j+1}=\frac{\mathrm{k}_{z, j}-\mathrm{k}_{z, j+1}}{\mathrm{k}_{z, j}+\mathrm{k}_{z, j+1}} e^{-2 k_{z, j} k_{z, j+1} \sigma_{j+1}^{2}}$ and $t_{j, j+1}=2 \mathrm{k}_{z, j} /\left(\mathrm{k}_{z, j}+\mathrm{k}_{z, j+1}\right)$, where $\sigma_{j+1}$ is the surface roughness of the $(j+1)$ th layer. The thicknesses of the MSTF and ITO layers are $h_{1}=$ $z_{0}-z_{1}$ and $h_{2}=z_{1}-z_{2}$, respectively. In this case, $R_{0}$ is the reflectivity coefficient of the sample. As shown in Fig. 3(a), the experimental XRR data are given along with the numerical simulation obtained using the expression for $R_{0}$ in Eq. (2). The data were fitted through the covariance matrix adaptation evolution strategy (CMA-ES) method. ${ }^{19,20}$ Since the wave functions, whose modulus describe EFI, are continuous at all the interfaces, the normalized EFI distribution can be simulated for the MSTF/ITO/glass system using the parameters obtained from the XRR fitting, as shown in Fig. 3(b). When the incident angle is less than the critical angle of silica, there are few X-rays that penetrate into the sample. The EFI is greatly enhanced in the MSTF layer when the incident angle is set between the critical angles of silica and ITO. When the incident angle is larger than the critical angle of ITO, the penetration depth of x-rays is strongly increased. As the incident angle increases, the scattering signal from ITO intensifies because $\mathrm{x}$-ray beam reaches deeper and excites more film volume. This is clearly manifested in Figs. 2(h) and 2(i), where the scattering from ITO dominates the central region of the GISAXS patterns and the Yoneda peaks of ITO (Y1) become evident. The first resonance mode $\left(\mathrm{TE}_{0}\right)$ of XSWs in the MSTF (the normalized EFI is $\sim 10$ ) emerges at the incident angle of $0.18^{\circ}$. This resonance mode presents as a dip on the XRR curve due to the increased absorption in the MSTF film, while the large interface roughness makes the dip shallower. The significance of the $\mathrm{TE}_{0}$ mode is further demonstrated by the GISAXS pattern shown in Fig. 2(d), where the diffuse scattering from the sample interfaces is substantially suppressed while the scattering signal from the MSTF film is strongly enhanced.

On the basis of the wave function analysis, the DWBA theory $^{21-24}$ is further employed to account for the multiple scattering effect and scattering from the mesochannels of MSTF. The scattering amplitude given as a function of the wave vector transfer $\mathbf{q}$ in this method ${ }^{25}$ is

$$
\begin{aligned}
A(\mathbf{q})= & T_{1, i} T_{1, f} F\left(q_{\|}, q_{1, z}\right)+T_{1, i} R_{1, f} F\left(q_{\|}, q_{2, z}\right) \\
& +R_{1, i} T_{1, f} F\left(q_{\|}, q_{3, z}\right)+R_{1, i} R_{1, f} F\left(q_{\|}, q_{4, z}\right),
\end{aligned}
$$

where $T_{1}$ and $R_{1}$ are the amplitudes of the reflected and transmitted X-ray beams in the MSTF, whose expressions are given in Eq. (2). The components of wave vector transfers are $\mathrm{q}_{\|}=\sqrt{\mathrm{q}_{x}^{2}+\mathrm{q}_{y}^{2}}, \quad \mathrm{q}_{1, z}=k_{z, f}-k_{z, i}, \quad \mathrm{q}_{2, z}=-k_{z, f}-k_{z, i}$, $\mathrm{q}_{3, z}=k_{z, f}+k_{z, i}$, and $\mathrm{q}_{4, z}=-k_{z, f}+k_{z, i}$, where $k_{z, i}$ and $k_{z, f}$ are the $\mathrm{z}$ components of the wave vectors of the incoming and outgoing x-ray beams given as $k_{z, i}=k_{0} \sqrt{n^{2}-\cos ^{2} \alpha_{i}}$ and $k_{z, f}=k_{0} \sqrt{n^{2}-\cos ^{2} \alpha_{f}}$, respectively. In these equations, $k_{0}=2 \pi / \lambda, \lambda$ is the $\mathrm{x}$-ray wavelength, $n$ is the refractive index, $\alpha_{i}$ is the incident angle, and $\alpha_{f}$ is the out-of-plane exit angle of the outgoing x-ray beam as shown in Fig. 1(a). The function $F$ is the form factor of the cylindrical mesochannels

$$
\begin{aligned}
F\left(q_{\|}, q_{z}, R, H\right) & =\left[2 \pi R^{2} \frac{J_{1}\left(q_{\|} R\right)}{q_{\|} R}\right] \times\left[H \operatorname{sinc}\left(q_{z} \frac{H}{2}\right) e^{-i q_{z} \frac{H}{2}}\right] \\
& =F_{\|\left(q_{\|}\right) \times F_{z}\left(q_{z}\right)}
\end{aligned}
$$

where $R$ and $H$ are the radius and height of the mesochannels. It can be separated into two independent functions of the in-plane $\left(q_{\|}\right)$and out-of-plane $\left(q_{z}\right)$ components of the wave vector transfer, denoted as $F_{\|}$and $F_{z}$. Since there is no long-range ordering of the mesochannels in MSTF, the inplane structure factor $S\left(q_{\|}\right)$can be simulated by the paracrystal model up to the second-order approximation ${ }^{26}$ 


$$
S\left(q_{\|}\right)=\frac{1}{N} \frac{1-\Phi\left(q_{\|}\right)^{2}}{1-2 \Phi\left(q_{\|}\right) \cos q_{\|} D+\Phi\left(q_{\|}\right)^{2}}-\frac{1}{N^{2}} \frac{2 \Phi\left(q_{\|}\left[\cos \left(q_{\|} D\right)-2 \Phi\left(q_{\|}\right)+\Phi\left(q_{\|}\right)^{2} \cos \left(q_{\|} D\right)\right]\right.}{\left[1-2 \Phi\left(q_{\|}\right)^{2} \cos \left(q_{\|} D\right)+\Phi\left(q_{\|}\right)^{2}\right]^{2}}
$$

where $\Phi\left(q_{\|}\right)=\exp \left(-\mathrm{q}_{\|}^{2} \delta_{D}^{2}\right), D$ is the average separation distance between the neighboring mesochannels with a dispersion width $\delta_{D}$, and $N$ is the number of mesochannels in the coherent scattering region. From Eqs. (3)-(5), we find that the expression for $\mathrm{x}$-ray scattering intensity can also be separated into two independent functions of $q_{\|}$and $q_{z}$, as given below

$$
\begin{aligned}
I_{\text {GISAXS }}\left(q_{\|}, q_{z}\right)= & {\left[\left|F_{\|}\left(q_{\|}\right)\right|^{2} S\left(q_{\|}\right)\right] } \\
& \times \mid T_{1, i} T_{1, f} F_{z}\left(q_{1, z}\right)+T_{1, i} R_{1, f} F_{z}\left(q_{2, z}\right) \\
& +R_{1, i} T_{1, f} F_{z}\left(q_{3, z}\right)+\left.R_{1, i} R_{1, f} F_{z}\left(q_{4, z}\right)\right|^{2} .
\end{aligned}
$$

In this way, the XSW-enhanced scattering effect that modulates the EFI distribution in MSTF couples only to the $q_{z}$ component of the form factor, and the correlation between
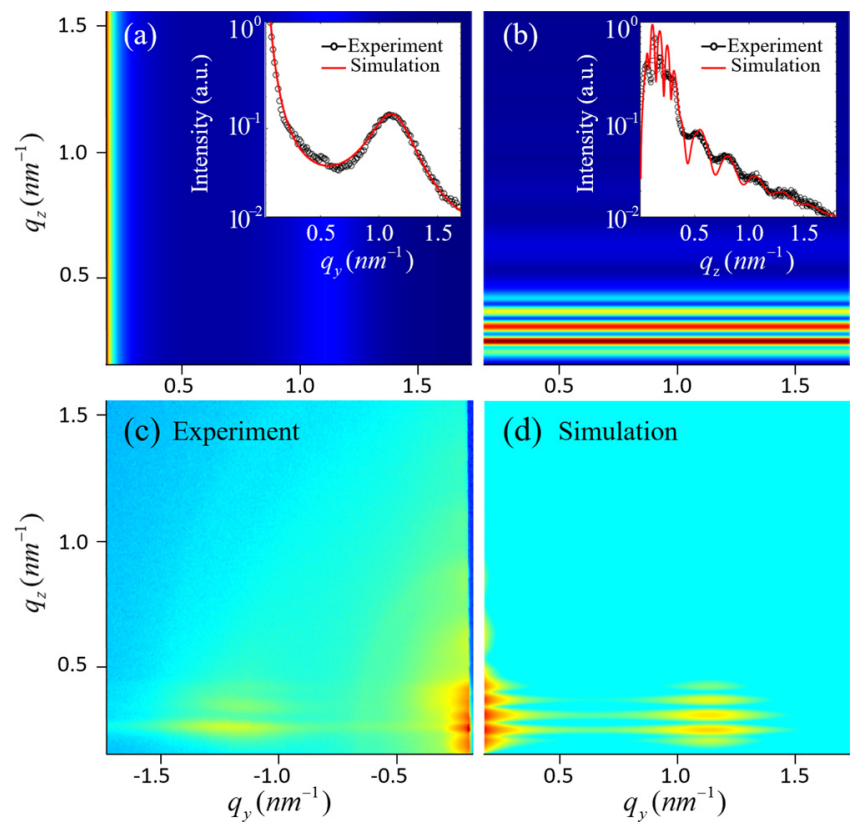

FIG. 4. (a) The simulated $q_{y}$-dependence of the GISAXS pattern. Inset: The experimental (black circles) and theoretical (red line) scattering intensity profiles extracted along the $q_{y}$ direction at $q_{z}=0.13 \mathrm{~nm}^{-1}$. (b) The simulated $q_{z}$-dependence of the GISAXS pattern. Inset: The experimental (black circles) and theoretical (red line) scattering intensity profiles extracted along the $q_{z}$ direction at $q_{y}=0.1 \mathrm{~nm}^{-1}$. (c) The experimental GISAXS pattern recorded at $\alpha_{i}=0.18^{\circ}$. (d) The simulated GISAXS pattern obtained by multiplying the data in (a) and (b).

TABLE I. The radius, $R$, and the height, $H$, and their standard deviations, $\sigma_{R}$ and $\sigma_{H}$, of the mesochannels; the average separation distance, $D$, and its dispersion width, $\delta_{D}$, of the neighboring mesochannels; and the number of mesochannels in the coherent scattering region, $N$ (all the units are in nm except $N$ ).

\begin{tabular}{lcccccc}
\hline \hline $\boldsymbol{R}$ & $\sigma_{\boldsymbol{R}}$ & $\boldsymbol{H}$ & $\sigma_{\boldsymbol{H}}$ & $\boldsymbol{D}$ & $\boldsymbol{\delta}_{\boldsymbol{D}}$ & $\boldsymbol{N}$ \\
\hline 2.21 & 0.32 & 29 & 0.7 & 5.35 & 0.23 & 14 \\
\hline \hline
\end{tabular}

neighboring mesochannels only affects the in-plane scattering intensity. Applying these results, the GISAXS data analysis becomes clear and tractable, in which the experimental data can be fitted separately in terms of $q_{\|}$and $q_{z}$. As shown in Fig. 4(a), the $q_{y}$-dependence of the GISAXS pattern is simulated using the in-plane component of Eq. (6), and the inset shows the scattering profile and the theoretical fitting along the $q_{y}$ direction for $q_{z}=0.13 \mathrm{~nm}^{-1}$. In the same way, the $q_{z^{-}}$ dependence of the scattering pattern calculated using the outof-plane component of Eq. (6) is shown in Fig. 4(b), where the inset is the experimental and theoretical curves along the $q_{z}$ direction extracted at $q_{y}=0.1 \mathrm{~nm}^{-1}$. Figure 4(c) shows the GISAXS pattern recorded at $\alpha_{i}=0.18^{\circ}$, and Fig. $4(\mathrm{~d})$ is the corresponding simulation result, which is the product of the data in Figs. 4(a) and 4(b), according to Eq. (6). As the EFI is distributed mainly at the center of the MSTF film, the diffuse scattering from the interface roughness is relatively weak and negligible. The structural parameters of MSTF obtained from the GISAXS data fitting are listed in Table I, where $\sigma_{R}$ and $\sigma_{H}$ are the standard deviations for $R$ and $H$. They agree well with those obtained from the SEM and TEM measurements. As can be seen by comparing the experimental and theoretical results in Figs. 4(c) and 4(d), the main scattering features are well replicated in the simulation employing the DWBA theory.

In conclusion, MSTFs are synthesized with their mesochannels aligned vertically to ITO glass substrates. X-ray scattering effects of the MSTF/ITO/glass system are comprehensively investigated by GISAXS and XRR techniques. XSWs are generated inside MSTFs even though the film void size and substrate roughness are several orders of magnitude larger than the incident x-ray wavelength. Good agreement is achieved between the experimental and theoretical GISAXS patterns and a complete picture of the dynamical scattering processes occurring in MSTFs is unveiled. The marked features of XSWs found in MSTFs would prompt new applications exploiting their unique properties beyond model films laid on ideal substrates. In view that $\mathrm{x}$-ray scattering from mesochannels is significantly enhanced by XSWs, the present findings should shed light on probing the requisite ionic and molecular transportation activities in mesoporous films engaged in fuel cell, battery and membrane separation applications.

See supplementary material for the GISAXS pattern of the MSTF film deposited on a bare glass substrate, the XRR fitting parameters, and the estimation of the critical angle of the MSTF film.

This work was supported by NSF of China (11375256, U1632265, and 21210004) and STC of Shanghai Municipality (14JC1493300 and 14JC1400700). We thank all the team members at BL16B and BL14B of SSRF. 
${ }^{1}$ U. Bach, D. Lupo, P. Comte, J. Moser, F. Weissörtel, J. Salbeck, H. Spreitzer, and M. Grätzel, Nature 395, 583 (1998).

${ }^{2}$ B. G. Trewyn, I. I. Slowing, S. Giri, H.-T. Chen, and V. S.-Y. Lin, Acc. Chem. Res. 40, 846 (2007).

${ }^{3}$ T. Wagner, S. Haffer, C. Weinberger, D. Klaus, and M. Tiemann, Chem. Soc. Rev. 42, 4036 (2013).

${ }^{4}$ W. Li, J. Liu, and D. Zhao, Nat. Rev. Mater. 1, 16023 (2016).

${ }^{5}$ K. C. Kao, C. H. Lin, T. Y. Chen, Y. H. Liu, and C. Y. Mou, J Am. Chem. Soc. 137, 3779 (2015).

${ }^{6}$ N. Linares, A. M. Silvestre-Albero, E. Serrano, J. Silvestre-Albero, and J. García-Martínez, Chem. Soc. Rev. 43, 7681 (2014).

${ }^{7}$ G. Renaud, R. Lazzari, and F. Leroy, Surf. Sci. Rep. 64, 255 (2009).

${ }^{8}$ J. Wang, M. J. Bedzyk, and M. Caffrey, Science 258, 775 (1992).

${ }^{9}$ M. J. Bedzyk, G. M. Bommarito, and J. S. Schildkraut, Phys. Rev. Lett. 62, 1376 (1989).

${ }^{10}$ S. Narayanan, D. R. Lee, R. S. Guico, S. K. Sinha, and J. Wang, Phys. Rev. Lett. 94, 145504 (2005).

${ }^{11}$ J. Wang, M. J. Bedzyk, T. L. Penner, and M. Caffrey, Nature 354, 377 (1991).

${ }^{12}$ J. A. Libera, R. W. Gurney, C. Schwartz, H. Jin, T. L. Lee, S. T. Nguyen, J. T. Hupp, and M. J. Bedzyk, J. Phys. Chem. B 109, 1441 (2005).

${ }^{13}$ M. J. Bedzyk, in Encyclopedia of Condensed Matter Physics, edited by G. L. Liedl and P. Wyder (Elsevier, Oxford, 2005), pp. 330.
${ }^{14}$ M. J. Bedzyk, in San Diego,' 91 , San Diego, CA (International Society for Optics and Photonics, 1991), p. 151.

${ }^{15}$ Z. Feng, C.-Y. Kim, J. W. Elam, Q. Ma, Z. Zhang, and M. J. Bedzyk, J. Am. Chem. Soc. 131, 18200 (2009).

${ }^{16}$ H. Kim, J. Horwitz, G. Kushto, A. Pique, Z. Kafafi, C. Gilmore, and D. Chrisey, J. Appl. Phys. 88, 6021 (2000).

${ }^{17}$ H. Kim, C. Gilmore, A. Pique, J. Horwitz, H. Mattoussi, H. Murata, Z. Kafafi, and D. Chrisey, J. Appl. Phys. 86, 6451 (1999).

${ }^{18}$ D. Jean and G. Alain, X-ray and Neutron Reflectivity: Principles and Applications (Springe r, Berlin, Heidelberg, 2009).

${ }^{19}$ N. Hansen and A. Ostermeier, Evol. Comput. 9, 159 (2001).

${ }^{20}$ G. Chen, P. H. Zwart, and D. Li, Phys. Rev. Lett. 110, 195501 (2013).

${ }^{21}$ S. K. Sinha, E. B. Sirota, S. Garoff, and H. B. Stanley, Phys. Rev. B 38, 2297 (1988).

${ }^{22}$ D. Babonneau, S. Camelio, D. Lantiat, L. Simonot, and A. Michel, Phys. Rev. B 80(15), 155446 (2009).

${ }^{23}$ Z. Jiang, D. R. Lee, S. Narayanan, J. Wang, and S. K. Sinha, Phys. Rev. B 84(7), 075440 (2011).

${ }^{24}$ S. V. Venkatakrishnan, J. Donatelli, D. Kumar, A. Sarje, S. K. Sinha, X. S. Li, and A. Hexemer, J. Appl. Crystallogr. 49(6), 1876 (2016).

${ }^{25}$ R. Lazzari, F. Leroy, and G. Renaud, Phys. Rev. B 76, 125411 (2007).

${ }^{26}$ X.-Q. Mu, Acta Crystallogr. Sect. A: Found. Crystallogr. 54, 606 (1998). 